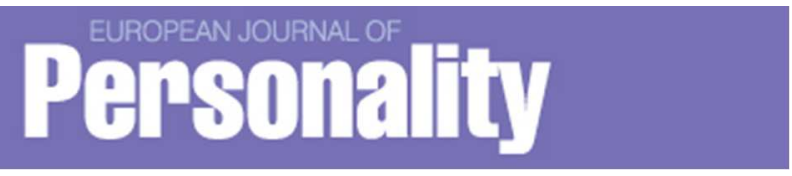

\title{
The Underlying Motives of Different Mixed-Motive Games
}

\begin{tabular}{|r|l|}
\hline Journal: & European Journal of Personality \\
\hline Manuscript ID & EJP-15-2034 \\
\hline Wiley - Manuscript type: & Response \\
\hline Date Submitted by the Author: & 16 -Sep-2015 \\
\hline Complete List of Authors: & $\begin{array}{l}\text { Haesevoets, Tessa; Ghent University, Department of Developmental, } \\
\text { Personality, and Social Psychology } \\
\text { Van Hiel, Alain; Ghent University, Department of Developmental, } \\
\text { Personality, and Social Psychology } \\
\text { Reinders Folmer, Chris; Erasmus University, Erasmus School of Law }\end{array}$ \\
\hline Manuscript Keywords: & \begin{tabular}{l} 
mixed-motive games, motivational basis, selfish choice, prosocial choice \\
\hline
\end{tabular} \\
\hline
\end{tabular}

SCHOLARONE ${ }^{m}$

Manuscripts 
Running head: MOTIVATIONAL BASIS OF MIXED-MOTIVE GAMES

The Underlying Motives of Different Mixed-Motive Games

\section{Tessa Haesevoets ${ }^{\mathrm{a}}$, Alain Van Hiel ${ }^{\mathrm{a}}$, and Chris Reinders Folmer ${ }^{\mathrm{b}}$}

${ }^{a}$ Ghent University; Department of Developmental, Personality, and Social Psychology; Henri Dunantlaan 2, B-9000, Ghent, Belgium

${ }^{b}$ Erasmus University; Erasmus School of Law; Burgemeester Oudlaan 50, 3062 PA, Rotterdam, The Netherlands

WORD COUNT: 4,315

\section{Authors' Note}

This research was supported by a research grant from the National Fund for Scientific Research-Flanders (Belgium) under grant number G095912N. Correspondence concerning this article should be addressed to Tessa Haesevoets, Department of Developmental, Personality, and Social Psychology, Henri Dunantlaan 2, B-9000, Ghent, Belgium. E-mail:

Tessa.Haesevoets@UGent.be, Tel.: ++32 (0)9 26491 41, Fax.: ++32 (0)9 2646499. 


\begin{abstract}
Haesevoets, Reinders Folmer, and Van Hiel (2015) have shown limited consistency of people's behaviour across various mixed-motive games. According to these authors, the modest relationships among these games call into question the general idea that all mixedmotive games render the conflict between selfish interests and concern for others equally salient. Thielmann, Böhm, and Hilbig (2015), however, argued that these findings can be explained in terms of the motivational differences that underlie the games. In this article, we demonstrate that Thielmann et al.'s descriptive model of the different motives underlying selfish and prosocial choices cannot be straightforwardly applied to the empirical data at hand. Analogous to our previous article, we again stress the need for further empirical research investigating the underlying motivational basis of each mixed-motive game.
\end{abstract} Keywords: mixed-motive games, motivational basis, selfish choice, prosocial choice 


\section{The Underlying Motives of Different Mixed-Motive Games}

In mixed-motive games people must choose between acting upon selfish interests (selfish choice) and concerns for others (prosocial choice). It is common practice within the field of psychology to accept that this self-other conflict is indeed a central characteristic of all mixed-motive situations (e.g., Dawes, 1980; Dawes \& Messick, 2000; Messick \& Brewer, 1983; Weber, Kopelman, \& Messick, 2004), and it is therefore generally expected that people's decisions are consistent across different games that are supposed to capture this conflict. Stated otherwise, when a person choses the prosocial option in one mixed-motive game, it can be reasonably expected that he or she will also choose this option in other mixedmotive games. The question that we (Haesevoets, Reinders Folmer, \& Van Hiel, 2015) tried to answer with our research was whether such generalizations are warranted.

In order to answer this question, we conducted a study $(N=219)$ in which choice behaviour across a wide range of different mixed-motive games was studied. Specifically, we included a Prisoner's Dilemma Game (PDG), an Assurance Game (AG), a Public Good Dilemma Game (PGDG), a Commons Dilemma Game (CDG), two Dictator Games (DG), an Ultimatum Bargaining Game (UBG), and a Trust Game (TG). Moreover, we also investigated the role of dispositional variables, that is, Social Value Orientation (SVO), Social Dominance Orientation (SDO), and Right-Wing Authoritarianism (RWA), which are considered to probe into the psychological conflict between concern for self and others.

Basically, the results of our study revealed a small average correlation across the different games $(r=.22, p=.001)$, which implies that behaviour in one mixed-motive game entails only little information about how someone will behave in another game. Further, a factor analysis revealed that the games loaded on two separate factors rather than on one common factor. Moreover, the dispositional variables that probe into concern for self versus others did not yield substantial behavioural effects in the different games as the average 
correlations among the dispositions and the games were small $(r=.16, p=.02 ; r=-.13, p=$ .06 ; and $r=-.14, p=.04$; for SVO, SDO, and RWA, respectively). Finally, the associations among the different games could not be explained by the dispositional variables. These findings led us to the conclusion that there is substantial variation in the behaviours exhibited in the various games, which suggests that conclusions drawn in one single game are not universal, and therefore may not extend to other games.

Thielmann, Böhm, and Hilbig (2015) wrote a comment on our paper. In the following, we will 1) evaluate this comment by comparing it to the content of our original manuscript, and 2) compare the predictions of Thielmann and colleagues' descriptive model to our actual data. Thereby, we aim to comprehensively evaluate the value of Thielmann et al.'s comment for understanding the results of our original manuscript.

\section{Motivational basis of different games}

Thielmann et al. (2015) stated on page 506 that our reasoning about the validity of mixed-motive games is rather pessimistic. We find it important to stress, however, that the word 'validity' was not used once in our manuscript. Indeed, the main aim of our paper was not to call into question the validity of the different games, but rather to investigate whether all games have a strong common ground. Therefore, we did not question the validity or importance of different games for understanding various social decisions; rather, our contribution questioned the practice of applying conclusions from specific games to mixedmotive decision making in general, or in specific other situations.

Further, Thielmann and colleagues (2015) argued that our findings can be interpreted in light of the variety of specific motives underlying decisions in different games. Here also, we think it important to refer to our original manuscript for clarification. Namely, our discussion of the findings explicitly underlined that "to have a better view on the unique characteristics of each mixed-motive game, future studies should examine these games in 
more detail with respect to their psychological basis and underlying motives. The study of games in greater detail in order to get a better view on its distinctive characteristics represents a key avenue for future research" (p. 454). Therefore, as the citation indicates, our own conclusions in fact already explicated the possibility that different motives operate in different games - a notion that unfortunately appears to have been overlooked in Thielmann et al.'s comment.

A more important issue relates to the question whether Thielmann and colleagues' (2015) descriptive model of the motives that underlie selfish versus prosocial choices in the different games (see Table 1, p. 507) is consistent with our data. It should be noted that Thielmann et al.'s model is based on an interpretation of the results of our study, and that no empirical data were collected. Although at first glance their proposed model seems promising, below we will argue that a comparison of its predictions with our actual data highlights several important discrepancies. We do not want to imply that their model is incorrect, but rather that these authors' statement that our findings "nicely mirror these motivational differences" (p. 507) is exaggerated.

In the remainder of this article we critically consider whether the magnitudes of the various correlations among games are in line with the proposed model. On page 507 of their comment, Thielmann et al. (2015) stated the following: "certain games should correlate rather strongly, as indeed they do in Haesevoets et al.'s data." Our analysis of the actual findings, however, questions whether this is indeed the case. For one, the correlations are typically too small to be considered as corroborative evidence for Thielmann et al.'s model. Specifically, the correlations among games which these authors considered to belong to the same game type are smaller than one would expect. Moreover, the analysis will show that games that are considered to have exactly the same motivational basis, do not yield very consistent behaviours either, which of course, is also at odds with the model. Not only the magnitudes of 
the correlations matter, but also the pattern of convergent and divergent relationships within and between game types. Here we will also note several inconsistencies between the hypothetical model and our empirical data. Another issue worthy of attention is that unlike the claim of Thielmann and colleagues, the PDG relates more strongly to the constant-sum game factor than to the social dilemma game factor, which neither corroborates the model. Finally, we will present additional data on the relationships between the HEXACO Agreeableness and Honesty-Humility dimensions and game behaviour. Contrary to the predictions of Thielmann and colleagues, in our data these personality dimensions are not strongly related to game behaviour.

\section{Do games cluster into constant-sum games and social dilemmas?}

Thielmann et al. (2015) made a distinction between two game types, that is, constantsum games and social dilemmas. In constant-sum games, the aggregated sum of players' payoffs is equivalent across choices, while in social dilemmas a prosocial choice increases the players' aggregated revenues. On the basis of this classification, it can be expected that the games that constitute one specific game type should correlate rather strongly with each other. However, this expectation is not confirmed by the data at hand. Indeed, as can be inferred from Table 2 (p. 451), the correlation between the two games that were categorized as constant-sum games, i.e., the DG and the UBG, was rather small in term of Cohen's (1988, 1992) conventions $(r=.17, p<.05)$. If indeed the constant-sum dimension constitutes the key dimension linking these two games, as suggested by Thielmann et al.'s typology, then one should expect a stronger association between both games.

Likewise, it can be expected that the correlations among the games that constitute the social dilemma game type should also be rather strong. We computed the average correlation among the five games that are classified as social dilemmas in Table 1 of Thielmann et al.

(2015, p. 507). After applying Fisher's $r$ to $z$ transformation (Fisher, 1921; as described in 
Howell, 1992), the average correlation among these five games just passed Cohen's threshold for being interpreted as moderate $(r=.31, p<.001)$.

Because in each of the mixed-motive games a combination of different motives shapes participants' choice for the prosocial or the selfish option, the expectation of a perfect correlation would of course be unrealistic, but it can nonetheless be concluded that the correlations obtained here are smaller than one might expect on the basis of the hypothesized strong motivational overlap that games within one particular game type are expected to share. Indeed, a closer look at Table 1 of Thielmann et al. (2015, p. 507) reveals that the two constant-sum games have the same underlying motivational basis (i.e., four motives are equal), with the exception that fear drives the prosocial choice in the UBG while this motive does not drive behaviour in the DG. Across the five social dilemmas, greed is the only motive that drives different behaviours. That is, greed drives the selfish choice in the PDG, the PGDG, and the CDG, but the prosocial choice in the AG and the TG. The six other reported motives are equivalent across the different social dilemmas. Hence, based on this rather strong common underlying motivational basis of the two constant-sum games and the five social dilemmas it is reasonable to expect stronger correlations within both game types than the ones found in our research.

If one follows the conventions of Cohen - as we explicitly did in our manuscript - a correlation coefficient greater than .50 can be interpreted as a large or strong association. According to this convention, the correlations that we obtained should never be called 'strong'. Even the highest correlation among our games fell short of this threshold. Indeed, as mentioned on page 449 , the behaviours shown in our games displayed rather small to moderate relationships, with five trivial $(r<.10)$, ten small $(.10<r<.30)$, and six moderate $(.30<r<.50)$ associations. It is therefore questionable whether the associations between the 
games indeed constitute rather strong associations, as Thielmann et al.'s (2015) framework predicts, and as these authors on page 507 concluded from our data.

\section{Behaviour across games with exactly the same motivational basis}

The relative weakness of the correlations among games is even better illustrated when looking at games that share the exact same underling motivational basis. For example, in Thielmann et al.'s Table (2015, p. 507) the AG and the TG have exactly the same underlying motivational basis as in both games the motives fairness, altruism, greed, trust, and social welfare drive the prosocial choice, while the motives fear and competitiveness drive the selfish choice. The observation that for these two games exactly the same motives underlie the different choices implies that the correlation between these two games should be very strong. However, a closer inspection of our correlation matrix (see Table 2, p. 451) shows that this is not the case. Indeed, our results revealed that the correlation between the AG and the TG can barely be interpreted as moderate $(r=.30, p<.001)$.

Similarly, according to Thielmann et al.'s Table (2015, p. 507) the PDG, the PGDG, and the CDG also have exactly the same underlying motivational basis. Here, the motives fairness, altruism, trust, and social welfare drive the prosocial choice, whereas the motives greed, fear, and competitiveness drive the selfish choice. However, after applying Fisher's $r$ to $z$ transformation, the average correlation between these three games turned out to be moderate $(r=.36, p<.001)$

In conclusion, in light of the common motivational basis that particular games are expected to share, these correlations can be considered to be rather modest. These results suggest that the overlap of the motivations underlying these games may be overestimated. Or, alternatively, that other distinctive motives beyond those identified in Thielmann et al.'s (2015) model are likely to shape decisions within the individual games. 


\section{Correlations within and between game types}

The classification of Thielmann et al. (2015) in terms of constant-sum games and social dilemmas also implies that games should display stronger correlations with games of the same game type than with games of the other game type. In other words, we consider the pattern of correlations rather than their sheer magnitude. Here too, a number of deviations from the model of Thielmann and colleagues can be noted.

For instance, the correlation between the DG and the UBG (which Thielmann et al., 2015, categorize as constant-sum games) should be stronger than, for instance, the correlation between the DG and the CDG (as the latter game is categorized as a social dilemma).

However, our correlation matrix (see Table 2, p. 451) indicates that this is not the case. Specifically, the correlation between the DG and the UBG $(r=.17, p<.05)$ was noticeably weaker (instead of stronger) than the correlation between the DG and the CDG $(r=.25, p<$ $.001)$. Although an updated version of Steiger's $Z$ test (Hoerger, 2013) revealed that the difference between these two correlations failed to reach statistical significance $\left(Z_{H}=-0.89, p\right.$ $=.38$ ), this result is nonetheless hard to reconcile with Thielmann and colleagues' model.

Additionally, within the social dilemma game type, the correlation between the AG and the TG (which share the exact same underling motives) should be stronger than their correlations with other social dilemmas that have a different motivational basis, like the PGDG in which greed underlies the selfish (instead of the prosocial) choice. However, the results of our study (see Table 2, p. 451) contradict this prediction as the correlation between the AG and the TG $(r=.30, p<.001)$ is about equal to or weaker (instead of stronger) than the correlation between the AG and the PGDG $\left(r=.28, p<.001 ; Z_{H}=0.3, p=.76\right)$ and between the TG and the PGDG $\left(r=.48, p<.001 ; Z_{H}=-2.49, p=.01\right)$.

In sum, we identified a number of patterns of convergent and divergent correlations within and between game types that are not consistent with Thielmann et al.'s (2015) 
presented model. These results thus further question the extent to which our findings can readily be understood in terms of the motivations advanced by Thielmann et al.

\section{Interpretation of factor structure}

In their comment, Thielmann and colleagues (2015) also noted that: "Considering this distinction between constant-sum games and social dilemmas, Haesevoets et al.'s finding of two separate game factors is both reasonable and consistent with game theory. In fact, in their study, constant-sum games and social dilemmas loaded on different factors” (p. 507). We also compared this interpretation with the actual data, which again indicated an important inconsistency with Thielmann and colleagues' typology.

Specifically, in the typology of Thielmann et al. (2015) the PDG is classified as a social dilemma, while our factor analysis (see Table 3, p. 451) revealed that the PDG loaded equally on both game factors. Moreover, a closer inspection of our correlation matrix (see Table 2, p. 451) shows that, in term of Cohen's conventions, the correlation between the PDG and the factor that constituted the so-called constant-sum games (i.e., Factor 2$)$ was large ( $r=$ $.60, p<.001)$, while the correlation between the PDG and the factor that constituted the socalled social dilemmas (i.e., Factor 1$)$ was only moderate $(r=.47, p<.001)$. The difference between these two correlation coefficients reached statistical significance $\left(Z_{H}=2.29, p=.02\right)$. Following the results of our study, the PDG should thus be categorized among the constantsum games instead of among the social dilemmas (contrary to the interpretation of Thielmann et al.).

Again, the actually data do not entirely fit the hypothesized structure of the theoretical model of Thielmann and colleagues (2015). Specifically, our analysis implies that the PDG should be categorized as a constant-sum game, while it has been asserted to be a social dilemma. 


\section{The HEXACO model of personality: Agreeableness and Honesty-Humility}

Finally, we reported that the dispositional variables that are considered to probe into the conflict between concern for self and others (i.e., SVO, SDO, and RWA) failed to consistently account for behavioural tendencies across games. In this regard, Thielmann and colleagues (2015) suggested that: "To account for behaviours in various games, one will need to consider a composition of personality traits capturing different pro-social tendencies (e.g. the HEXACO model of personality)” (p. 508). In light of further clarification, we want to mention that this point was also raised during the review process. In fact, based on the reviewer comments we collected new data and also included the HEXACO personality dimensions Agreeableness and Honesty-Humility, as recent research found that particularly Honesty-Humility (and to a lesser extent Agreeableness) is associated with cooperative behaviour (see Hilbig \& Zettler, 2009; Hilbig, Zettler, \& Heydasch, 2012; Hilbig, Zettler, Leist, \& Heydasch, 2013). In our cover letter sent to the action editor on December 12th, 2014, we reported the findings regarding these HEXACO personality dimensions. Specifically, in light of their limited contribution, we argued that it was not necessary to include the results of these dimensions. However, it does mean that we can provide conclusive evidence of these relationships here.

The present Table 1 reports the correlations between Agreeableness and HonestyHumility and the seven mixed-motive games. In terms of Cohen's conventions, these relationships were all trivial or small ( 8 out of 14 non-significant), with an exception for the moderate correlation between Honesty-Humility and the DG $(r=.33, p<.001)$. For both personality dimensions, we calculated the average correlation with the seven mixed-motive games (after applying Fisher's $r$ to $z$ transformation). The average correlation between Agreeableness and the seven games as well as the average correlation between HonestyHumility and the seven games was small and non-significant (both $r \mathrm{~s}=.11, p \mathrm{~s}=.10$ ). Hence, 
Agreeableness and Honesty-Humility both explain on average only $1.21 \%$ of the variance in game behaviour.

Next, we investigated whether the shared variance among the different games and the factor scores can be explained by Agreeableness and Honesty-Humility. Therefore, we computed the correlations among the games and the factor scores, while controlling for these two personality dimensions. The results of this partial correlation analysis are also displayed in Table 1 (see the values between the brackets). The 26 significant correlations all remained significant after controlling for Agreeableness and Honesty-Humility. Subsequently, we also investigated whether the correlation coefficients of the zero-order correlation (in which we did not control for personality) and the correlation coefficients of the partial correlation (in which we did control for personality) significantly differed from each other. These analyses revealed that for none of the 26 correlations such a difference occurred (all $\Delta r \mathrm{~s}<.09, p \mathrm{~s}>$ $.19)$.

In sum, other than the suggestion made by Thielmann et al., the personality dimensions Agreeableness and Honesty-Humility are only modestly related to different behaviours in the various games.

\section{Conclusion}

The comment by Thielmann and colleagues (2015) further explored the (shared or distinct) motivational differences that may underlie decisions in different mixed-motive games. Certainly, we believe that an analysis of the motivational basis of games is valuable for understanding these games. However, with regard to the descriptive model proposed by Thielmann et al., the present analysis has indicated important discrepancies with our actual findings, which question the extent to which our findings indeed "are actually perfectly in line with the game-theoretic understanding of the different economic games" (p. 506) and "nicely mirror these motivational differences" (p. 507). 
First of all, the magnitudes of the correlations among games within the broad game types of constant-sum games and social dilemmas are too small to be considered as corroborative evidence for Thielmann and colleagues' (2015) descriptive model. Similarly, the correlations among the games with exactly the same motivational basis were much smaller than expected. Moreover, we identified a number of patterns of convergent and divergent correlations within and between game types which are also inconsistent with Thielmann et al.'s model. Finally, in the typology of Thielmann and colleagues the PDG was classified as a social dilemma, which is also hard to reconcile with the empirical data at hand. Our analysis thus has indicated important discrepancies from the model proposed by Thielmann et al. While the motives that they suggest certainly may play a role in explaining decisions in individual games, the modest associations between - according to their model - highly similar games question the conclusiveness of their explanation.

Hence, our results only partially (rather than perfectly) reflect the motivational differences as proposed by Thielmann and colleagues (2015). Moreover, the results presented above suggest that there might be other important motives in play which were not included in Thielmann et al.'s framework. Rather than relying on game-theoretic abstractions and generalizations, our analysis underlines the importance to fully understand the unique basis of individual mixed-motive games, and the psychological processes associated with each of them. As stated in the discussion section of our original paper, we believe that to get a better view on the unique characteristics of each mixed-motive game, future empirical studies should examine each mixed-motive games in more detail with respect to its psychological basis and underlying motives (e.g., Van Lange, Liebrand, \& Kulhman, 1990; Van Hiel, Vanneste, \& De Cremer, 2008). 


\section{REFERENCES}

Cohen, J. (1988). Statistical Power Analysis for the Behavioral Sciences. Lawrence Erlbaum Associates.

Cohen, J. (1992). A power primer. Psychological Bulletin, 122, 155-159.

Dawes, R. M. (1980). Social Dilemmas. Annual Review of Psychology, 31, 69-93.

Dawes, R. M, \& Messick, D. M. (2000). Social dilemmas. International Journal of Psychology, 35, 111-116.

Fisher, R. A. (1921). On the probable error of a coefficient of correlation deduced from a small sample. Metron, 1, 3-32.

Haesevoets, T., Reinders Folmer, C., \& Van Hiel, A. (2015). Cooperation in mixed-motive games: The role of individual differences in selfish and social orientation. European Journal of Personality, 29, 445-458.

Hilbig, B. E., \& Zettler, I. (2009). Pillars of cooperation: Honesty-Humility, social value orientations, and economic behavior. Journal of Research in Personality, 43, 516-519.

Hilbig, B. E., Zettler, I., \& Heydasch, T. (2012). Personality, punishment and public goods: Strategic shifts towards cooperation as a matter of dispositional honesty-humility. European Journal of Personality, 26, 245-254.

Hilbig, B. E., Zettler, I., Leist, F., \& Heydasch, T. (2013). It takes two: Honesty-Humility and Agreeableness differentially predict active versus reactive cooperation. Personality and Individual Differences, 54, 598-603.

Hoerger, M. (2013). $Z_{H}$ : An updated version of Steiger's $Z$ and web-based calculator for testing the statistical significance of the difference between dependent correlations. Retrieved from http://www.psychmike.com/dependent_correlations.php.

Howell, D. C. (1992). Statistical methods for psychology (3rd ed.). Belmont, CA: Duxbury Press. 
Messick, D. M., \& Brewer, K. (1983). Solving social dilemmas: A Review. In L. Wheeler, \& P. Shaver (Eds), Review of personality and social psychology. Beverly Hills, CA: Sage Publications, Inc.

Thielmann, I., Böhm, R., \& Hilbig, B. E. (2015). Different Games for Different Motives: Comment on Haesevoets, Folmer, and Van Hiel (2015). European Journal of Personality, 29, 506-508.

Van Hiel, A., Vanneste, S., \& De Cremer, D. (2008). Why did they claim too much, or too little? The role of causal attributions in explaining level of cooperation in commons and anticommons dilemmas. Journal of Applied Social Psychology, 38, 173-197.

Van Lange, P. A. M., Liebrand, W. B. G., \& Kulhman, D. M. (1990). Causal attribution of choice behavior in three N-person Prisoner's dilemma's. Journal of Experimental Social Psychology, 26, 34-48.

Weber, M., Kopelman, S., \& Messick, D. (2004). A conceptual review of decision making in social dilemmas: Applying the logic of appropriateness. Personality and Social Psychology Review, 8, 281-307. 


\section{TABLE 1.}

Correlation matrix for the personality dimensions Agreeableness and Honesty-Humility (Pearson's $r$ ).

\begin{tabular}{|c|c|c|c|c|c|c|c|c|c|c|}
\hline Variable & 1. & 2. & 3. & 4. & 5. & 6. & 7. & 8. & 9. & 10. \\
\hline 1. Agreeableness & - & & & & & & & & & \\
\hline 2. Honesty-Humility & $.22^{* *}$ & - & & & & & & & & \\
\hline 3. PDG & $.14^{*}$ & $.15^{*}$ & - & & & & & & & \\
\hline 4. $A G$ & .12 & -.00 & $.10(.09)$ & & & & & & & \\
\hline 5. PGDG & $.13^{*}$ & .09 & $.37^{* * *}\left(.35^{* * *}\right)$ & $.28^{* * *}\left(.27^{* * *}\right)$ & - & & & & & \\
\hline 6. $\mathrm{CDG}$ & .10 & .12 & $.27^{* * *}\left(.25^{* * *}\right)$ & $.34^{* * *}\left(.34^{* * *}\right)$ & $.44^{* * *}\left(.43^{* * *}\right)$ & - & & & & \\
\hline 7. DG & $.15^{*}$ & $.33^{* * *}$ & $.29^{* * *}\left(.25^{* * *}\right)$ & $.15^{*}\left(.15^{*}\right)$ & $.26^{* * *}\left(.23^{* * *}\right)$ & $25^{* * *}\left(.22^{* *}\right)$ & - & & & \\
\hline 8. UBG & -.00 & .05 & $.07(.06)$ & $-.04(-.04)$ & $-.01(-.01)$ & $.06(.06)$ & $.17^{*}\left(.17^{*}\right)$ & - & & \\
\hline 9. TG & $.16^{*}$ & .05 & $.19^{* *}\left(.17^{*}\right)$ & $.30^{* * *}\left(.29^{* * *}\right)$ & $.48^{* * *}\left(.47^{* * *}\right)$ & $.33^{* * *}\left(.32^{* * *}\right)$ & $.13(.11)$ & $.03(.03)$ & - & \\
\hline 10. Factor 1 & $.19^{* *}$ & .13 & $.47^{* * *}\left(.45^{* * *}\right)$ & $.51^{* * *}\left(.50^{* * *}\right)$ & $.87^{* * *}\left(.86^{* * *}\right)$ & $.68^{* * *}\left(.67^{* * *}\right)$ & $.36^{* * *}\left(.33^{* * *}\right)$ & $.01(.01)$ & $.72^{* * *}\left(.72^{* * *}\right)$ & - \\
\hline 11. Factor 2 & $.15^{*}$ & $.31^{* * *}$ & $.60^{* * *}\left(.58^{* * *}\right)$ & $.11(.11)$ & $.37^{* * *}\left(.36^{* * *}\right)$ & $.40^{* * *}\left(.38^{* * *}\right)$ & $.89^{* * *}\left(.87^{* * *}\right)$ & $.38^{* * *}\left(.39^{* * *}\right)$ & $.07(.04)$ & $.45^{* * *}\left(.43^{* * *}\right)$ \\
\hline
\end{tabular}

Note. $\mathrm{PDG}=$ Prisoner's Dilemma Game, $\mathrm{AG}=$ Assurance Game, $\mathrm{PGDG}=$ Public Good Dilemma Game, CDG $=$ Commons Dilemma Game, DG $=$ Dictator Game, UBG = Ultimatum Bargaining Game, and TG = Trust Game. The Dictator Game represents the mean standardized score of two Dictator Games. For the Commons Dilemma Game, the sign of the correlations were adjusted (because a high score indicated defection). The values between the brackets represent the correlations when controlling for Agreeableness and Honesty-Humility. ${ }^{*} p<.05,{ }^{* *} p<.01,{ }^{* * *} p<.001$. 